Polymer Journal, Vol. 39, No. 1, pp. 48-54 (2007)

(C)2006 The Society of Polymer Science, Japan

\title{
Role of Stearic Acid in Preparing EPDM/Clay Nanocomposites by Melt Compounding
}

\author{
Yong MA, Qi-Fang LI, Li-Qun ZHANG, and You-Ping $\mathrm{WU}^{\dagger}$ \\ Key laboratory for Nanomaterials of Ministry of Education, Key Laboratory of Beijing City \\ for Preparation and Processing of Novel Polymer Materials, \\ Beijing University of Chemical Technology, Beijing 100029, China
}

(Received July 31, 2006; Accepted October 19, 2006; Published December 1, 2006)

\begin{abstract}
EPDM/organic clay (OC) nanocomposites were prepared by melt blending. XRD results showed that if OC mixed with EPDM matrix, macromolecule chains are difficult to intercalate into the layers while stearic acid (SA) could easily intercalate into the OC interlayers under pretreating conditions (S-OC) and expand the interlayer spacing $2.6 \mathrm{~nm}$ of OC to a wider distance of $3.9 \mathrm{~nm}$; EPDM could not further expand the distance of S-OC during the followed mixing process and the structure SA intercalating into OC is kept. TEM observation showed S-OC improved the dispersion of OC in EPDM, and FT-IR results indicated the esterification reaction occurred between carboxyl groups of SA and hydroxyl groups of OC, which was believed to be the driving force of SA intercalation. The tensile strengths of the nanocomposites are improved with increasing amount of SA, and the stresses at low strain are almost the same, due to the physical interaction between OC and EPDM macromolecules. [doi:10.1295/polymj.PJ2006085] KEY WORDS Organoclay / Rubber / Stearic Acid / Intercalation / Nanocomposites /
\end{abstract}

Since the researchers at Toyota central research laboratories successful prepared PA6/clay nanocomposites for the first time, ${ }^{1}$ as a new kind of materials, polymer/clay nanocomposite have caught much attention through the world. Compared with pure materials and traditional composites, polymer/clay nanocomposites exhibit increased modulus and strength, improved heat resistance, outstanding gas barrier properties, and decreased flammability. ${ }^{2-4}$

For rubbers/layer nanocomposite, there are mainly three kinds of preparation methods: latex, ${ }^{5}$ solution $^{6}$ and melt compounding. ${ }^{7}$

Considering industrialization, melt compounding is one of the feasible preparation techniques, in which polymer is directly intercalated into organoclay (OC) in the molten state to prepare nanocomposites. The driving force of the intercalation is determined by physical and/or chemical interactions between polymers and OC.

At the same time, as one of the widely used and fast growing synthetic rubbers, Ethylene-propylene-diene terpolymer (EPDM) finds applications in automotive weather-strapping and seals, radiator, electrical insulation, roofing membrane, tubing, belts, and other general-purpose applications because of its excellent resistance to heat, oxidation, ozone, weathering, and microbial attack, attributed to the stable and saturated polymer backbone structure. ${ }^{8}$ This motivated us to develop rubber/clay nanocomposites based on EPDM.

For nonpolar saturated EPDM matrix, the functional oligomers, with polar telechelic groups as a compa- tibilizer, e.g., EPDM grafted by maleic anhydride ${ }^{9-11}$ or glycidyl methacrylate, ${ }^{12}$ were used to improve the interfacial interaction. In addition, there are hydroxyl groups $^{13,14}$ on the surface of clay layer, carboxylic acids have been used to intercalate into clay gallery to modify the clay. ${ }^{1,15}$

Stearic acid (SA), usually used as a kind of additives for rubber sulfur vulcanization, is an acidic ingredient like maleic anhydride, and can react with the hydroxyl groups on the surface of clay layer, which should contribute to improving the intercalation of SA. In this paper, the effects of SA on the intercalation and dispersion of OC in EPDM were investigated by mixing SA-pretreated OC with EPDM to produce rubber/clay nanocomposites. According to the experimental results, SA could easily intercalate into OC interlayers and be esterified with hydroxy groups on OC surface. Shortly, using SA pretreated OC in melt blending method is a possible processing technique to prepare nanocomposite with outstanding dispersion.

\section{EXPERIMENTAL}

\section{Materials}

EPDM, J2080, with 67\% ethylene and 6\% ethylidene norbornene content having a Mooney viscosity of 86 at $100^{\circ} \mathrm{C}$, was purchased from Jilin Chemical Industrial Co. Ltd. (China); OC intercalated by Dimethyl Dialkyl (C14-18) Ammonium (Nanomer I.44 P) with $65 \%$ montmorillonite was supplied by

${ }^{\dagger}$ To whom correspondence should be addressed (Tel: +86-10-6443-4860, Fax: +86-10-6443-3964, E-mail: wuyp@ mail.buct.edu.cn). 
Nanocor Inc., USA. Other compounding ingredients, including zinc oxide ( $\mathrm{ZnO}), \mathrm{SA}$, and sulfur (S), and tetramethyl thiuram tetrasulfide (TMTD) were commercial grade products.

\section{Preparation of Pretreated OC with SA}

SA and OC were mixed in homomixer for about $1 \mathrm{~min}$ at room temperature. The mixture was put in an oven at $100^{\circ} \mathrm{C}$ (the melting point of SA is nearly $70^{\circ} \mathrm{C}$ ) for about $1 \mathrm{~h}$ to ensure that melted SA reacted with OC and intercalated into the interlayers of OC. After cooling, OC pretreated with SA was obtained, which was denoted as S-OC.

\section{Preparation of OC/EPDM Nanocomposites}

EPDM and S-OC were mixed on an open two-roll mill at room temperature for about $10 \mathrm{~min}$. TMTD, $\mathrm{ZnO}$ and $\mathrm{S}$ were added to obtain the compounds; then the compounds were vulcanized in a standard mold for $\mathrm{t}_{90}$ at $160^{\circ} \mathrm{C}$ under the pressure of $15 \mathrm{MPa}$. Disk rheometer P355B2 produced by Beijing Huanfeng Chemical Industry Machine Experiment Factory (China) was used to measure optimum cure time $\left(t_{90}\right)$.

The same amount of OC, SA and other additives were directly mixed with EPDM on an open two-roll mill to prepare the contrast samples.

The base recipe is EPDM $100 \mathrm{phr}$, TMTD $1.2 \mathrm{phr}$, $\mathrm{ZnO} 5 \mathrm{phr}$ and $\mathrm{S} 1.5 \mathrm{phr}$; SA is variable (2 phr, $5 \mathrm{phr}$ and $10 \mathrm{phr}$ ).

\section{Characterization}

X-Ray diffraction (XRD) of OC, S-OC and OC/ EPDM nanocomposites was carried out by using a diffractometer (D/Max-III C, Rigaku, Japan) with $\mathrm{CuK} \alpha$ radiation operating at $40 \mathrm{kV}$ and $200 \mathrm{~mA}$; the range of $2 \theta$ is from $0.5^{\circ}$ to $10^{\circ}$ at a scan rate of $1^{\circ} / \mathrm{min}$.

Transmission electron microscopy (TEM) observations were performed on the ultrathin sections with an H-800 TEM (Hitachi, Japan) at an acceleration voltage of $200 \mathrm{kV}$ at room temperature, and the ultrathin sections were prepared with a cryoultramicrotome under liquid nitrogen cooling.

Fourier transform infrared spectroscopic (FT-IR) measurements were also used to investigate the possible interaction between SA and OC. FT-IR on the films was performed at a resolution of $4 \mathrm{~cm}^{-1}$ by using a Hitachi 270-30 spectrometer (Hitachi, Japan). $\mathrm{OC}, \mathrm{S}-\mathrm{OC}$ and SA were pressed with $\mathrm{KBr}$ powder, respectively, for FT-IR measurements in the transmission mode.

Mechanical properties tests were carried out on CMT4104 electric tensile tester (SANS, Shenzhen China) at a crosshead speed of $500 \mathrm{~mm} / \mathrm{min}$ and $23{ }^{\circ} \mathrm{C}$ according to ASTM D 412 method, and the stress-strain curves were drawn simultaneously.

\section{RESULTS AND DISCUSSION}

\section{FT-IR Analysis}

FT-IR spectrums of OC, SA and S-OC at the ratio 2:10 of SA to OC are shown in Figure 1. According to Figure 1a, there are no peaks corresponding to $\mathrm{C}=\mathrm{O}$ structures, which is consistent with the structure of OC. The negative peak at $1706 \mathrm{~cm}^{-1}$ in Figure 1b refers to the $\mathrm{C}=\mathrm{O}$ structure of carboxylic groups in SA. For S-OC, the negative peak is shifted to $1726 \mathrm{~cm}^{-1}$, which means that carboxylic groups are esterified (Figure 3c) by hydroxy groups of OC layers. This esterification between SA and OC can be the driving force for SA intercalating into $\mathrm{OC}$ to expand the spacing of OC.

\section{XRD Analysis}

XRD spectrograms of OC, compounds and nanocomposites are presented in Figure 2. The diffraction peak on XRD spectrogram of $\mathrm{OC}$ can be found at $2.3^{\circ}(2.6 \mathrm{~nm}$, curve a). When OC is directly added to EPDM matrix, the interlayer distance is just expanded to $2.9 \mathrm{~nm}$ (curve b) and the peak became broader, which means that EPDM molecule chains intercalate into interlayers of OC to some degree. After $\mathrm{SA}$ is added to OC/EPDM mixture, the spacing is dramatically expanded to $3.9 \mathrm{~nm}$ (curve c), indicating that $\mathrm{OC}$ is obviously intercalated. Additionally, the peaks at $4.4^{\circ}$ of $2 \theta$ (marked as $*$ ) can be observed, which are looked as secondary diffraction peaks and related to reflections at higher order. ${ }^{10}$ If the X-ray reflects from the third layers of clay, the parameter $n$ in Bragg Law $(n \lambda=2 d \sin \theta)$ should be 2 , and distance of the peak is the same as the first peak. The appearance of secondary diffraction peaks indicates that the clay layers exist at higher order of arrangement. In fact, scarce of adequate orientation of clay layers, secondary diffraction peaks seldom appear when testing OC powder (curve a) or the system with worse dispersion of OC (curve b).

To clarify which is intercalated into interlayers, SA or EPDM, XRD analysis of S-OC at the ratio of SA to $\mathrm{OC}$ of 2:10 is further carried out, and the spectrograms are also presented in Figure 2.

The interlayer distance of S-OC is shifted from $2.6 \mathrm{~nm}$ of $\mathrm{OC}$ to $3.9 \mathrm{~nm}$ (curve d), which is the same as that in curve c. After adding S-OC to EPDM matrix, the layer distance remains unchanged (curve e), indicating that the expanding space should be attributed to SA intercalation. It is consistent with the above results of FT-IR. SA can intercalate into the interlayers of OC more easily than EPDM, and the reasons can be the following: (1) during mixing process, long EPDM chains have much higher viscosity than SA, 


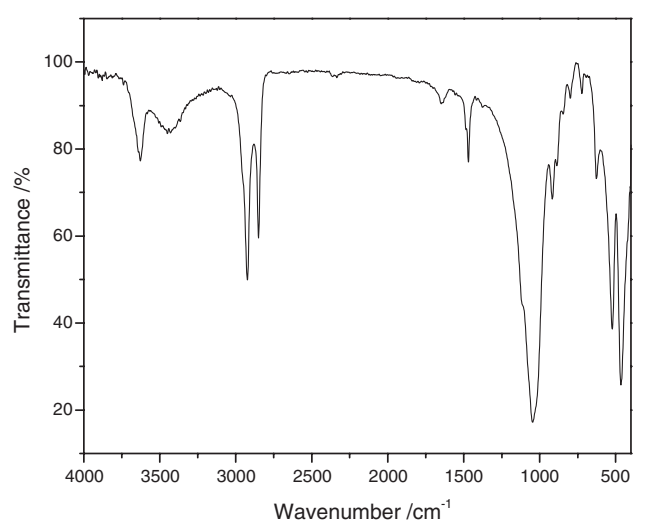

(a)

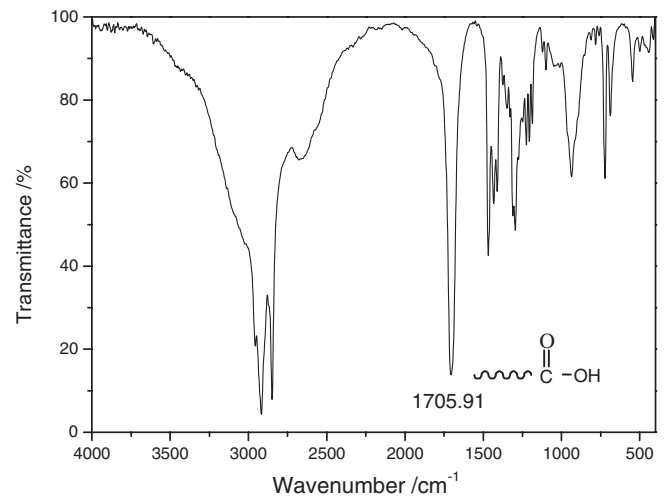

(b)

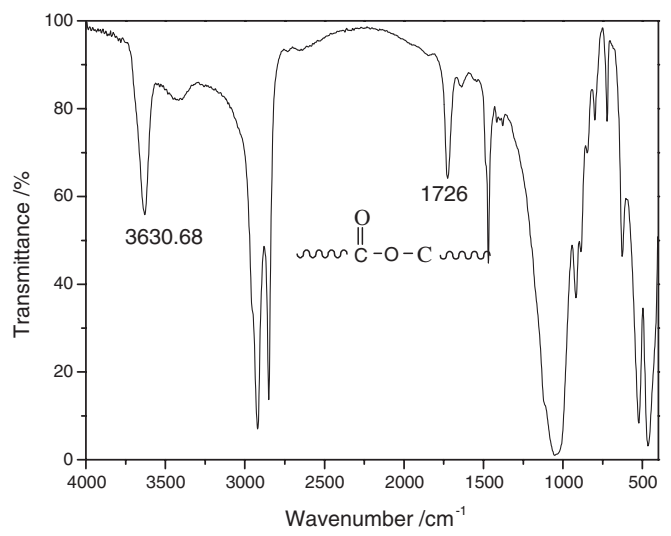

(c)

Figure 1. FT-IR spectrogram of (a) OC, (b) SA and (c) S-OC at the ratio 2:10 of SA to OC.

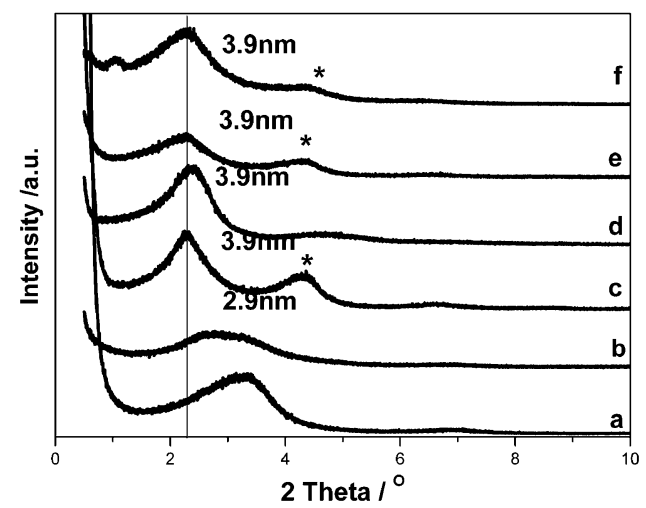

Figure 2. XRD spectrograms of OC, S-OC, compounds and nanocomposites at the ratio 2:10 of SA to OC: $a, O C ; b, O C /$ EPDM compound; c, after adding SA into OC/EPDM compound; d, S-OC; e, after adding S-OC into EPDM; f, vulcanizate of sample $f$ with other ingredients added.

and thus the mobility of EPDM molecules is relatively lower; whereas the mixture of OC and SA was kept for $1 \mathrm{~h}$ at $100^{\circ} \mathrm{C}$ (higher than the melting point of $\mathrm{SA}, 70^{\circ} \mathrm{C}$ ) during the pretreating process, at which SA is in melting state and the viscosity is low due to the low molecule weight, and the mobility of SA molecules is higher than that of EPDM; (2) interac- tions between nonpolar saturated EPDM macromolecule and OC are weaker than that between polar SA and OC; (3) as shown by FT-IR results, the esterification between carboxylic groups on SA and hydroxy groups on OC layers also provided the driving force for SA intercalation into OC.

S-OCs are powders, and layers could not be orderly arrayed, so secondary diffraction peaks could not be observed (curve d). If the amount of SA is high enough to form continuous phase and clay layer be mobile, secondary diffraction peaks could be observed, which will be discussed later.

Additionally, after adding S-OC into EPDM, the intensity of diffraction peak decreases and the width of peak is broader (curve e) relative to S-OC. When adding S-OC to matrix, some EPDM macromolecule chains intercalate into the edge of layers, disturbing the arrangement of the parallel layers and decreasing the interaction between clay layers. Under the shearing force, some clay is exfoliated to smaller aggregates or single layers with SA on the surface, leading to the decrease in intensity of diffraction peak. At the same time, during mixing process, the small aggregates can agglomerate to form incompact agglomerates. EPDM chains between exfoliated single layers 
are also kept between the interlayers, and form wider clearance, resulting in the broader diffraction peak.

After curing, intensity of diffraction peak increases, which suggests that more orderly intercalated structures are formed (curve f). At the curing temperature of $160{ }^{\circ} \mathrm{C}$, the viscosity of rubber matrix decreases, and pressure makes intercalated aggregates arrange more orderly. ${ }^{18}$

Additionally, for vulcanizate, a small peak appears at low angle, which may be related to the reaction between SA, ZnO and modifier. (1) During the process of curing, SA and $\mathrm{ZnO}$ can react to produce zinc stearate. Therefore some SA molecules which are absorbed on the surface of exfoliated clay layers will be desorbed, and ammonium modifier can be exposed. (2) $\mathrm{Zn}^{2+}$ can complex with amine group of modifier, ${ }^{17-19}$ and exposed ammonium modifier is easier to complex with $\mathrm{Zn}^{2+}$, which may drag the exfoliated layer to closer distance leading to the appearance of the peak at lower angle due to the existence of some EPDM molecules.

As discussed above, SA could easily intercalate into the interlayers of S-OC. To further investigate the intercalation of SA into OC, the ratio 10:10 of SA to OC was studied, and the XRD spectrums are shown in Figure 3. XRD spectrum of SA is also shown in Figure 3 to discern the effect of the crystal peak of SA. The diffraction peak become sharper and more asymmetric for S-OC (curve b), and sharp part of diffraction peak in curve $b$ is just like crystal diffraction peak of SA (curve a), suggesting that the sharp part is the result of crystal peak of SA. Since the content of SA is higher, some SA molecules do not participate in the esterification reaction, and recrystallize during the cooling process. Moreover, SA molecules form

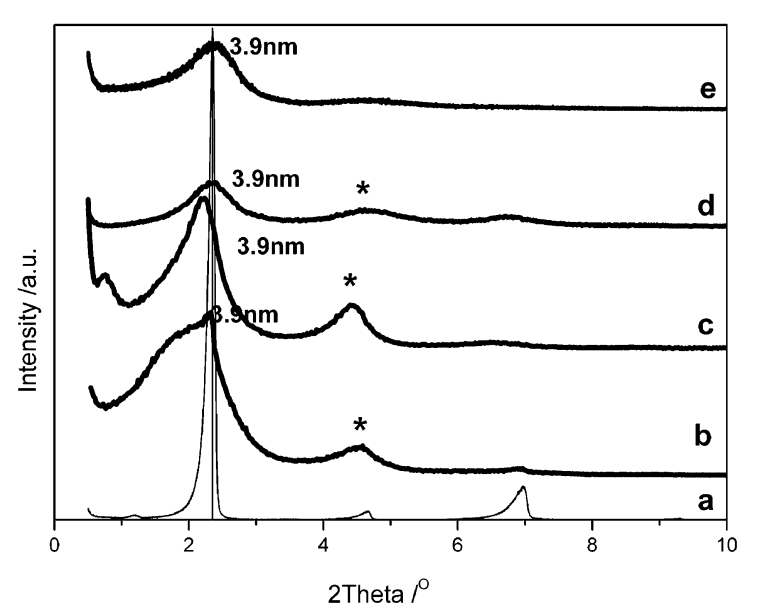

Figure 3. XRD spectrograms of SA, S-OC, compounds and nanocomposites: a, SA; b, S-OC at the ratio 10:10 of SA to OC; c, after adding S-OC into EPDM; d, vulcanizate of sample $\mathrm{c}$ with other ingredients added. continuous phases at the ration 10:10 of SA to OC, clay layer have adequate mobility, secondary diffraction peaks (marked as $*$ ) is observed clearly even in S-OC powder.

After S-OC was added to EPDM matrix, the concentration of SA dramatically decreased, and in the meantime the crystal was destroyed during mixing process. These led to the disappearance of the crystal diffraction peak of SA (Figure 3c).

Another phenomenon, which should be noticed, is that the intensity of diffraction peak decreases after vulcanization (curve d), which is completely different from the case at the ratio 2:10 of SA to OC (curve $g$ in Figure 2).

At the ratio 10:10 of SA to OC, there is some free SA in EPDM matrix, and thus SA absorbed by exfoliated clay layers could not desorbed to participate in curing reaction during vulcanization. At $160^{\circ} \mathrm{C}$ of vulcanizing temperature, the macromolecule chains with lower viscosity can enter into some of interlayers easily, and exfoliate the clay layers from the aggregates or divide aggregates into smaller ones, and some free SA existing in EPDM matrix can be quickly absorbed on the new surface of clay layers so that the curing pressure could not force the aggregation of clay layers. Therefore, the dispersion of S-OC is improved during curing process, and the intensity of diffraction peak weakens.

Additionally, the minor peak at lower angle in Figure $2 \mathrm{f}$ does not appear in Figure $3 \mathrm{~d}$, which is related to the fact that SA could not be desorbed during the curing process and shield the complex action of ammonium modifiers with $\mathrm{Zn}^{2+}$.

\section{TEM Observation}

In order to further make sure of the OC dispersion, TEM observations were performed. The results are shown in Figure 4.

If OC is directly added to EPDM matrix, clay layers do not disperse well, and aggregates can be observed (Figure 4a); adding SA, the aggregates disperse to much smaller ones, or even to some thinner layers (Figure $4 b$ ). When S-OC is added into EPDM matrix, the dispersion is greatly improved. The lamellas are SA/OC intercalated aggregates, not single clay layers (Figure 4c). With more SA loadings, thinner lamellas can be observed in Figure 4d. Besides, many blurred filaments can be observed, which are those exfoliated smaller aggregates and even single clay layers.

Absence of enough driving force, EPDM macromolecules cannot intercalate into the interlayers of clay, and large number of OC aggregates exist in the matrix. Adding SA, SA intercalated into OC and decreased the interactions between layers, and the dispersion is improved to some degree. However, in EPDM ma- 


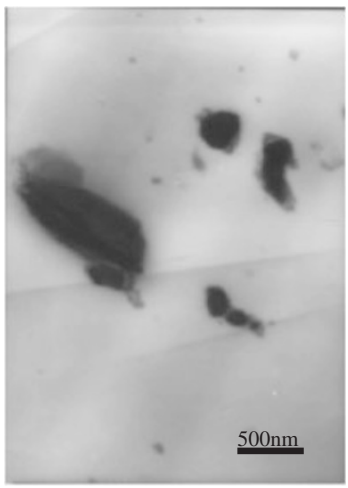

a, EPDM/OC

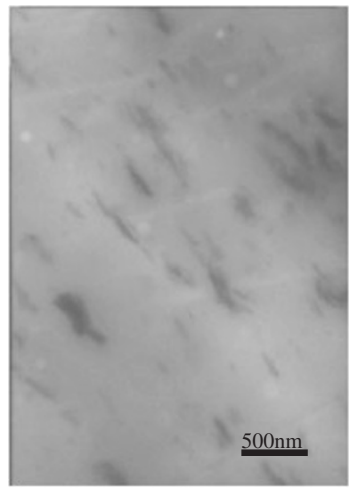

c, EPDM/S-OC $(\mathrm{SA}: \mathrm{OC}=2: 10)$

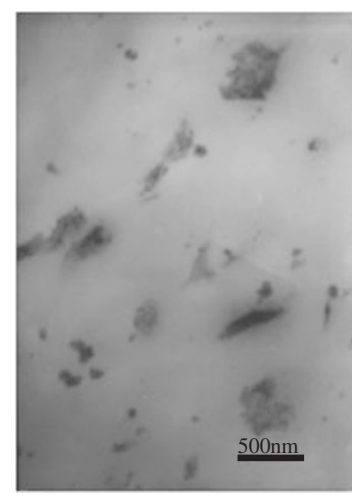

b, EPDM/OC/SA(SA:OC=2:10)

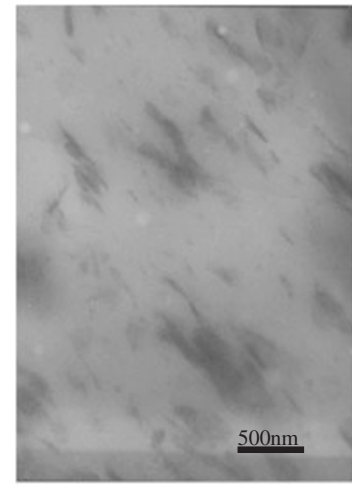

d, EPDM/S-OC $(\mathrm{SA}: \mathrm{OC}=10: 10)$
Figure 4. TEM photos of EPDM/OC and EPDM/S-OC compounds.

trix, the probability that SA and OC meet and react is much lower than in S-OC, and the dispersion is not as good as using S-OC. When increasing the amount of SA, more SA chains intercalate into the interlayers of OC, and separate the aggregates to smaller blocks and quickly pack the clay layers or aggregates. With the distance expanding, the interaction between layers is dramatically weakened. Under shearing force, some EPDM macromolecule chains are intercalated into the edge of layers, which promoted the exfoliation of S-OC. Aggregates become smaller lamellas or single layers, that is why many blurred filaments can be observed only in Figure 4d.

Considering XRD and TEM analysis, we can describe the evolved structure of OC in the different systems in Figure 5. In OC, clay layers (thick straight line) are orderly arrayed with modifier chains (filament) absorbed on the surface and form aggregates. Mixing OC with rubber matrix, the interlayers are slightly expanded. Lacking of strong interactions, only few EPDM chains (light colored line) can enter into the edge of interlayers and some clay layers are just forced to slightly incline. Then adding SA (broken line), the esterification between carboxylic groups on SA and hydroxy groups on OC layers can help SA intercalate into the clay interlayer. Due to low concen- tration of SA and $\mathrm{OC}$ in matrix (e.g., when the ratio of SA to OC is $2: 10$, the concentration of SA and OC in the compound is about $2 /(2+10+100)=$ $1.8 \%$ and $10 /(2+10+100)=8.9 \%$, respectively $)$, and only parts of interlayers are intercalated by SA chain.

Pretreating OC with SA, OC and SA both have higher concentrations (e.g., if the ratio of SA to OC is $2: 10$, the concentrations of SA and OC in S-OC is $2 /(2+10)=16.7 \%$ and $10 /(2+10)=83.3 \%$, respectively), and the reaction probability between SA and OC is increased, and most of SA chains can intercalate into the clay layers. Although the interlayer distance is expanded, the layers still agglomerate as aggregates. Adding S-OC to matrix, the aggregates are exfoliated to single layers or divided into smaller ones. For the unexfoliated part, there are only SA chains between the interlayers, and the interlayer distance is not changed. In the system with less SA loadings, clay layers mainly exist as smaller aggregates (Figure 5e) but with more SA loadings, single clay layers are more easily observed (Figure 5f). What's more, there are many free SA (dark colored line) dispersed in the matrix.

According to XRD and TEM results, the genuine structure in these systems should be: single layers and smaller aggregates of SA/OC intercalating structure dispersed in matrix.

\section{Mechanical Properties Analysis}

To farther clarify the effect of SA on dispersion of clay layers, mechanical properties of OC/EPDM (the contrast sample with $2 \mathrm{phr}$ SA) and S-OC/EPDM samples with different SA loadings are carried on and stress-strain curves are shown in Figure 6.

From Figure 6, with more SA loadings, the tensile strength of the contrast sample is only $3.4 \mathrm{MPa}$ (a), but for the samples using S-OC, the tensile strengths dramatically enhance from $4.3 \mathrm{MPa}$ of $2 \mathrm{phr}$ SA (b) to $7.7 \mathrm{MPa}$ of $5 \mathrm{phr} \mathrm{SA}$ (c), and reach up to 20.1 MPa of $10 \mathrm{phr}$ SA (d). This is related to the dispersion of clay. During the tensile process, the silicate layers will be arrayed to improve the orientation of macromolecules, and better tensile properties of nanocomposites can be obtained regardless of the function of compatibilizer. ${ }^{20}$ Obviously, in those systems, single clay layers or thin layers aggregates are more, and the tensile strengths are better.

The stresses at low strains of those samples using OC or S-OC with different SA loadings are not obviously different and the curves are almost the same. It is attributed to the fact that the interfacial interaction between OC and EPDM matrix is mainly physical. Adding SA can increase the dispersion of OC, but can not strengthen the interfacial interaction. 


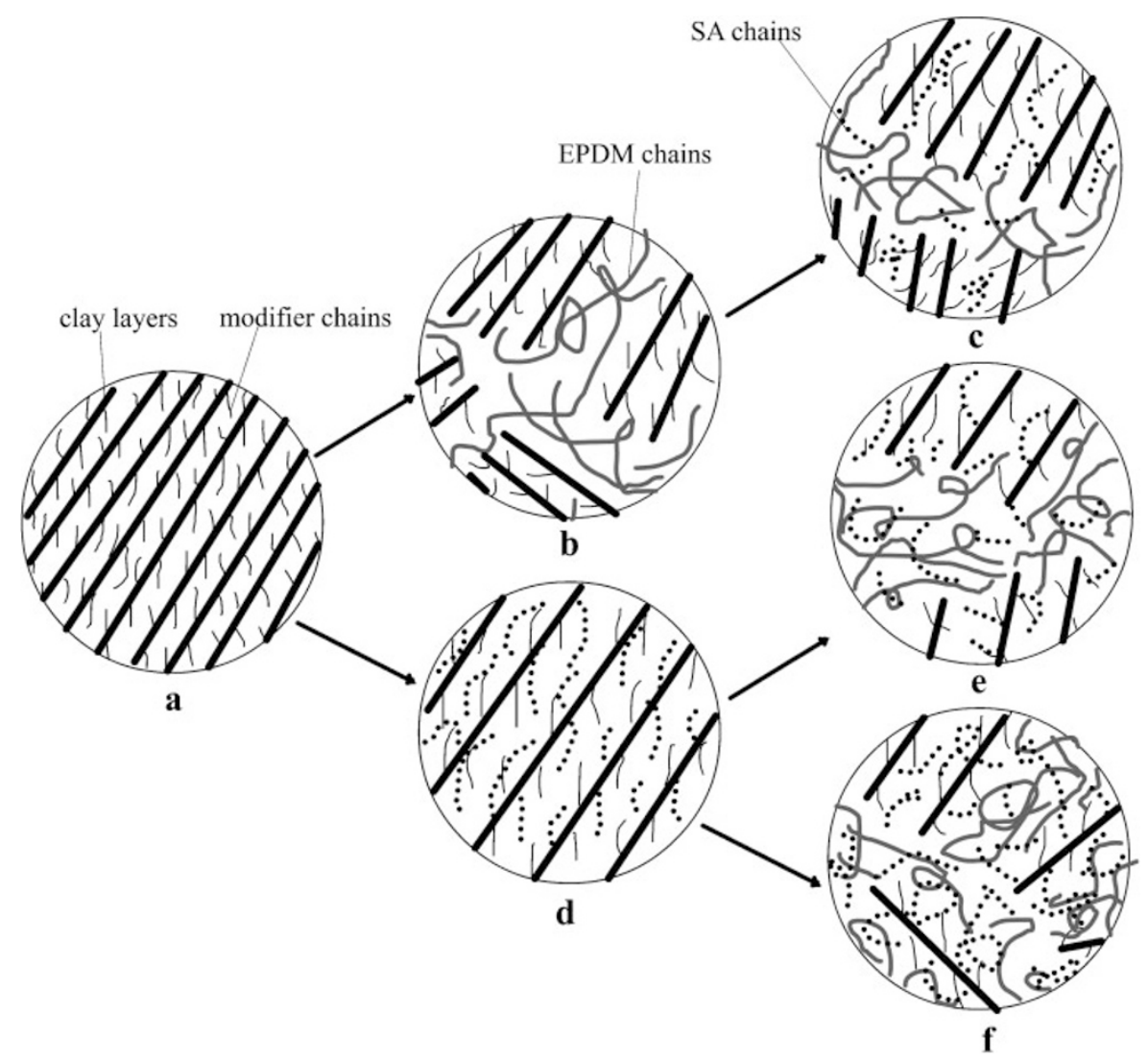

Figure 5. The sketch of evolved structure of OC: a, OC; b, OC/EPDM compound; c, after adding SA into OC/EPDM compound; d, S-OC; e, after adding S-OC (SA:OC $=2: 10$ in S-OC) into EPDM; f, after adding S-OC (SA:OC = 10:10 in S-OC) into EPDM.

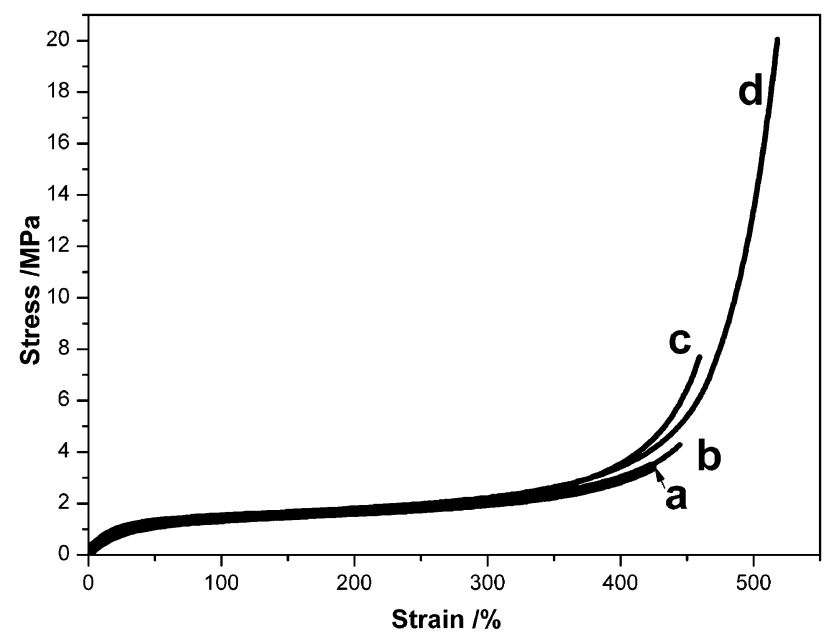

Figure 6. Stress-strain curves of OC/EPDM and S-OC/ EPDM nanocomposites: a, the contrast sample (SA 2 phr); b, 2 phr $\mathrm{SA}$ in S-OC; c, 5 phr SA in S-OC; d, 10 phr SA in S-OC.

\section{CONCLUSION}

SA can intercalate into the interlayers of OC and be esterified with hydroxy groups on the surface of clay, which is the driving force of intercalation for SA. With the help of shearing force, S-OC can be divided into thinner aggregates or even exfoliated during mix- ing process, but the intercalation structure with the distance of $3.9 \mathrm{~nm}$ in these systems is only formed by SA and OC. With increasing SA amount, the dispersion of S-OC in EPDM was increased.

Changing the fillers from OC to S-OC and increasing the amount of SA from $2 \mathrm{phr}, 5 \mathrm{phr}$ to $10 \mathrm{phr}$, and the tensile strengths of the nanocomposites are obviously improved, which is related to the better dispersion of clay. However, the stresses at low strain of those samples are nearly the same, because SA cannot reinforce the interaction between OC and EPDM macromolecules.

Acknowledgment. The authors gratefully acknowledge the National Tenth-five Program (grant number: 2001BA310A12), the Key Project of Beijing Natural Science Foundation (grant number: 2031001), and the financial support of the Beijing New Star Plan Project (grant number: 2004A14).

\section{REFERENCES}

1. A. Usuki, Y. Kojima, M. Kawasumi, A. Okada, Y. Fukushima, T. Kurauchi, and O. Kamigaito, J. Mater. Res., 8, 1179 (1993).

2. A. Mousa and J. Karger-Kocsis, Macromol. Mater. Eng., 286, 260 (2001). 
3. M. Zanetti, S. Lomakin, and G. Camino, Macromol. Mater. Eng., 279, 1 (2000).

4. Y. Q. Wang, H. F. Zhang, Y. P. Wu, and L. Q. Zhang, J. Appl. Polym. Sci., 96, 318 (2005).

5. Y. P. Wu, Q. X. Jia, Y. Q. Wang, and L. Q. Zhang, J. Appl. Polym. Sci., 89, 3855 (2003).

6. M. Pramanik, S. Srivastava, B. K. Samantaray, and A. K. Bhowmic, J. Appl. Polym. Sci., 87, 2216 (2003).

7. S. Varghese and J. Karger-Kocsis, J. Appl. Polym. Sci., 91, 813 (2004).

8. J. Karger-Kocsis, A. Kallo, A. Szafner, G. Bodor, and Z. S. Senyei, Polymer, 20, 37 (1979).

9. A. Usuki, A. Tukigas, and M. Kato, Polymer, 43, 2185 (2002).

10. K. G. Gatos and J. Karger-Kocsis, Polymer, 46, 3069 (2005).

11. H. Zheng, Y. Zhang, Z. Peng, and Y. Zhang, J. Appl. Polym. Sci., 92, 638 (2004).

12. K. G. Gatos, R. Thomann, and J. Karger-Kocsis, Polym. Int.,
53, 1191 (2004).

13. C. Chow, M. Chiang, and J. Lin, Macromol. Rapid Commun., 26, 1841 (2005).

14. J. Lin J, Y. Chang, and I. Cheng, Macromol. Rapid Commun., 25, 508 (2004).

15. H. Byun, M. Choi, and I. Chung, Chem. Mater., 13, 4221 (2001).

16. Y. R. Liang, Y. L. Lu, Y. P. Wu, Y. Ma, and L. Q. Zhang, Macromol. Rapid Commun., 26, 926 (2005).

17. P. J. Nieuwenhuizen, A. W. Ehlers, J. W. Hofstraat, S. R. Janse, M. W. F. Nielen, J. Reedijk, and E.-J. Baerends, Chem. Eur. J., 4, 1816 (1998).

18. C. P. Reyneke-Barnard, M. H. S. Gradwell, and W. J. Mcgill, J. Appl. Polym. Sci., 77, 2732 (2000).

19. A. Dirksen, P. J. Nieuwenhuizen, M. Hoogenraad, J. G. Haasnoot, and J. Reedijk, J. Appl. Polym. Sci., 79, 1074 (2001).

20. Y. Ma, Y. Wu, Y. Wang, and L. Zhang, J. Appl. Polym. Sci., 99, 914 (2006). 